\title{
Image Recognition System using Geometric Matching and Contour Detection
}

\author{
Gaurav Sharma \\ Deptt. of Electronics \& \\ Communication Engg. \\ Lovely Professional University \\ Jalandhar, India
}

\author{
Sonali Sood \\ Deptt. of Electronics \& \\ Communication Engg. \\ Lovely Professional University \\ Jalandhar, India
}

\author{
Gurjot Singh Gaba \\ Deptt. of Electronics \& \\ Communication Engg. \\ Lovely Professional University \\ Jalandhar, India
}

\author{
Nancy Gupta \\ Deptt. of Electronics \& Communication Engg. \\ CT Institute of Engineering and Management \\ Shahpur Campus Jalandhar
}

\begin{abstract}
Image recognition plays an important role in a wide range of applications from biomedical imaging and security systems to scene surveillance and many other fields. This document presents the representation of the recognition of two images through the process of geometric comparison. The geometric comparison is performed by comparing the image with a template through the processes of edge detection, scaling, contour matching and RGB to grayscale conversion. The software utilized is NI vision which is one of applications of the National Instrument's LabVIEW, used for the image and video processing functions
\end{abstract}

\section{General Terms}

Image recognition, Geometric matching, Contour detection, Template extraction.

\section{Keywords}

Image recognition, Edge detection, Contour matching, Score matching, Virtual Instrument (VI), Region of Interest (ROI).

\section{INTRODUCTION}

The ability of the human vision is excellent to match the perceived images with the already imbibed images in the brain. Moreover, the ability to imbibe perceived images as new ones in the memory, relating them with the other senses like touch, hearing, smell or taste is awesome [1]. These human senses are extraordinary, but have certain limitations of being tired and being not so fast as the computer vision. Computer vision aids the image recognition using the projection of a group of projects in parts of collection which is easily formable and linkable [2]. But care must be taken that the images must be robustly match able and recognized with ignorance to external factors which are unimportant [3]. As such, use of the computer vision for building a system for the image recognition would be helpful. Hence the image recognition system has been created using the National Instruments' LabVIEW.

National Instruments's LabVIEW is system design software that provides the developers an easy and effective way to deploy the hardware and system integration. It encompasses the tools capable of providing high level of the hardware integration. It finds a very wide outreach in the fields of research and development, ranging from the data acquisition to logic implementation to instrument control and creation of virtual hardware environments in a real-time computer system [4].

One such application of the NI LabVIEW is NI Vision, used for the image and video processing functions. This software provides hardware and software tools for image acquisition and processing to address applications such as quality and process control, automated testing for semiconductor, automotive and electronics, intelligent monitoring, and medical imaging. This aids in the vital processes like biomedical imaging, thermal image processing and various other applications including the facial recognition systems.

The research work deals with the image recognition of any image under test with a live template created. The template would be used for comparison with the image under test and thus locating the area of interest in the image. It will scan the whole image for finding the availability of template area in the file under testing, the number of instances available in the picture and the location of the images present in it. The supported image format is JPEG format, in the grayscale mode. The process first requires to create the template which has to be compared with the test object. The image comparison requires the use of NI Vision tools. The IMAQ (Image Acquisition) tools are used to acquire, capture and display the images as well as to configure the hardware according to our logical requirements. It allows the acquiring of images from the file systems as well as from real time sources [5].

\section{RELATED WORK}

Though significant amount of image recognition systems have been developed in the past using various platforms, there has been a very few instances of development of such systems in the NI LabVIEW. The important contributions regarding this field are discussed under this context.

Buhmann et al. in [1] have described the original process of the human vision to recognize the images and to correlate them with the other senses. The important features of the process are the Contour detection, edge detection, geometric comparison and color matching which are vital in being used for the artificial recognition systems.

Felzenszwalb and Huttenlocher in [2] have used the pictorial segmentation of the various parts of an image to find the instances of the parts in the images. These deformable 
parts are able to trace out the instances of the segmented parts in the image.

Lowe in [3] has developed the distinctive image extraction pattern method to extract the invariant features for matching with the huge number of the images in the image database through methods like varied illumination, added noise, affine distortion etc.

Johnson and Jennings in [4] have described the usage of the LabVIEW environment for the various hardware and software interfacings and relating the virtual environment with real environment.

Klinger in [5] has highlighted the use of IMAQ Vision in the applications of image processing. Moreover, he discussed the use of NI Vision tools and the library virtual instrument's (VI's) in the field of image processing.

Park et al. in [6] have used the histogram equalization technique for the image refinement. This helps in the better feature detection of the image and aids in a better comparison. This improves the contrast of the image and hence, the image features become more distinct.

Shan Du in [7] has described the feature extraction of an image. He described the use of suitable algorithms to extract the various features of the image like edges, contours etc. the features can be used for robust image recognition systems.

Sandal and Iyengar in [8] have conducted the research over the recognition of the human faces and their expressions under various conditions. These help in generating various situational algorithms for the facial structure of the human face matching as well as for the matching of images in real time systems.

Chellapa et al. in [9] have compared the human as well as machine image recognition process. They have basically outlined the main processes where the human vision recognition differs from the machine counterpart. This helps in formulating a system methodology for the development of the recognition systems.

Bartlett et al. in [10] have used the independent component analysis for the facial recognition systems. This includes the feature measurements of each of the facial components of the human face. These are then used for the matching of the already stored images in the template database.

\section{TEMPLATE EXTRACTION}

The template is the foremost part of the working of the project. A template has to be extracted from a real sample source for the comparison with the image under test. The template is the basic platform of comparison and hence, the following have to be kept in mind while choosing a base image for template extraction:

\subsection{Choose a clear image:}

The base image chosen for the template extraction must be a good quality image with proper edges, preferably from a high resolution camera [9]. Real time camera systems generally do not provide high quality images; hence taking images with a good quality digital camera is preferred.

\subsection{Prefer grayscale images:}

The image comparison works on the grayscale matching [10]. Hence, preferring a grayscale image for the template facilitates in providing better extraction as well as better outputs.

\subsection{Prefer images on a single coloured background:}

Images with single colored backgrounds are easy for extraction of the facial curves. Try to choose an image which has a light background. This facilitates in the auto ignoring of the inclusion of the background contours into the template. An image with the white background gave the best results.

\subsection{Try to choose images with a plain face expression:}

As the process works on the geometric matching of the edges and features, the images with plain expressions on the face must be preferred which are more general [8] [9] [10]. Special expressions may result in the error generation during the comparison.

The software used for the extraction of the templates from the images is the NI template creator. This software enables the user to input any supported file format and extract the template image out of it for comparison. Here, the file is input, the curves are defined and the areas of interest are included in the curves. The curves represent the actual comparison area of the image. The area covered by these images is used for comparison.

Once the image has been chosen, the area of interest has to be defined from the image. This should be the face of the person whose template is to be defined. It should be noted that the area of the template must be chosen in such a way that it includes the most of the portion of the face and ignores the rest of the body. Neck portions may be included but during the conduction of the proposed work, the images included with the neck gave inaccurate results.

The auto template extraction usually extracts the outer edges of the image. For the contour and other features of the image, the user has to define the curves on the additional areas like eyes, nose, lips etc. For an enhanced comparison, the areas like the eyeball structures and the ears can be included in the comparison. But as the size of the outlines of these features changes too rapidly as compared to the other features, it is usually advisable to avoid comparing these features into the base template.

Fig. 1 shows the template extraction of a facial image. The red drawn curves are the ones which highlight the prominent facial features of the image. It may be noted that the features which are less prominent have been ignored out of the system.

Once the template has been extracted, careful inspection of the resulting curves of the image should be carried out. Any left out features or any extra curve being included in the template should also be checked. The extra regions can be ignored by using the option of regions to ignore. These regions would not be included in the resulting curves. After the template has been finalized, save it as a template file. It is usually saved as a Portable Network Graphics (PNG) file. The templates have to be extracted for each of the images to be compared. 


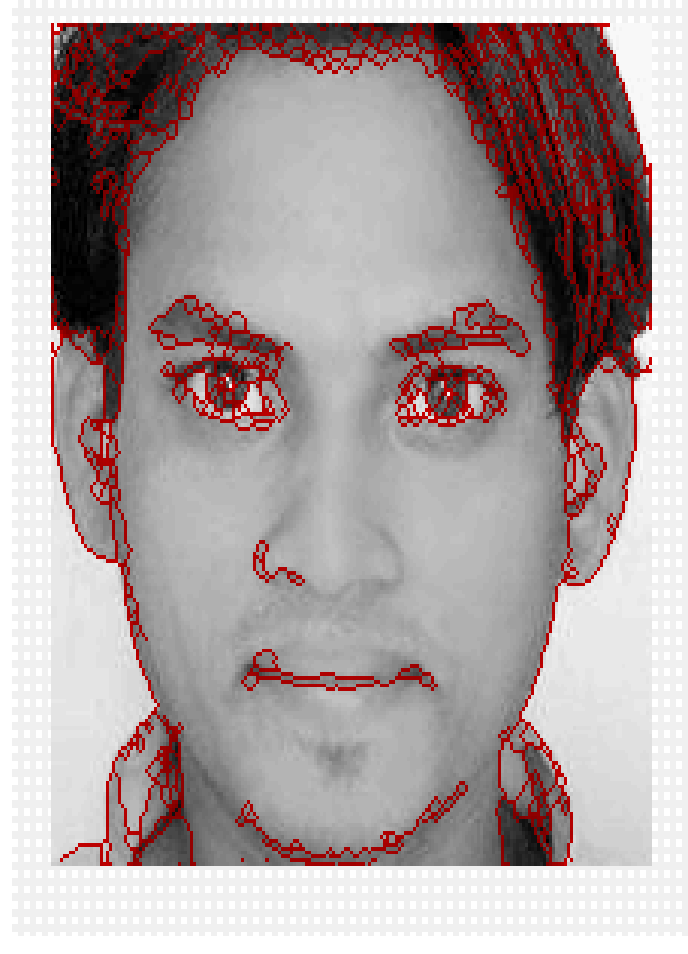

Fig 1: Template extraction of the highlighted features of the image.

\section{IMAGE COMPARISON}

This is the part of the system that deals with the matching of the image under test with the database templates already extracted. For the image comparison, the images chosen are preferably JPG formats. The currently developed system does not support files of the other formats than JPEG and PNG files.

The feature based matching of the images facilitates the virtual instruments (VI's) to compare the image with a template and find out the features in the image, their location as well as the number of exact matches. A grayscale image is used for the purpose.

$\log (x, y)=\frac{-1}{\pi \sigma^{4}}\left[1-\frac{x^{2}+y^{2}}{2 \sigma^{2}}\right] e^{-\frac{x^{2}+y^{2}}{2 \sigma^{2}}}$

Equation (1) represents the logical equation for the edge matching algorithm of the image. The $(\mathrm{x}, \mathrm{y})$ represent the coordinates of the particular pixel of the image. $\sigma$ represents the standard Gaussian Deviation. The above coordinate equation works when the images are centered to zero.

The whole work carried out is based on the following algorithm stated here under. The algorithm clearly specifies the basic processes such as creation, extraction of template, comparison and ROI processes.

Image comparison requires the use of advanced tools of the NI Visions. These are the virtual instruments used for the construction of the major system that consists of the necessary blocks in a systematic way. The main VI is a combination of further sub-VI's that construct the system.
Algorithm 1: Image recognition using edge detection and contour matching.

While Termination $\neq 1$;

Create Blank Image and Template Space.

Input Template \& Image;

If image $=$ Valid;

If Yes,Goto label 1;

If No, End;

label 1;

Input Score ${ }_{\text {match }}$;

Input Image Match Filters Viz Scaling, Rotation, Ignore Colour etc.

Extract Image Features using equation (1);

If Scaling = 1;

Check Image \& template size;

If Size $_{\text {image }}=$ Size $_{\text {Template; }}$

Scale Image to Template using scaling feature;

Else Set Size $=$ default;

If $\operatorname{Rotation}=1$;

Rotate Image coordinates;

Else Image coordinates $=(0,0)$;

Set Match coordinates = origin;

For $\mathrm{I}=0$ to $\mathbf{P}_{\mathbf{i}}$.

$$
\begin{aligned}
& \text { If Pixel }{ }_{\text {template }}=\text { Pixel }_{\text {Image; }} \\
& \text { Score }=\text { score }+1 ;
\end{aligned}
$$

end;

Match $_{\text {out }}=($ Score $/ \mathrm{Pi}) * 1000$;

If Match $_{\text {out }}=$ Score $_{\text {Match; }}$

Initialize ROI;

Draw ROI in 0 to $\mathrm{Pi}$;

Output ROI;

end;

else end;

Output Match ROI;

Display Image;

Output Score;

Dispose Template and Image Space;

end;

end while; 


\subsection{Blank Image Space Formation:}

The first part is the creation of the blank image spaces for the testing purpose. This includes the path definition of the files. The user has been given the option of choosing the file template and the folder of the collection of image database collection. The VI would search the database and thus result out the image which matches the template. A region of interest (ROI) is marked around the matched face and thus would indicate the detection.

\subsection{Learning The Geometric Patterns:}

After the image input, the IMAQ image tools are used to learn the template region. For this, the learn geometric function of the IMAQ library is used. This function enables the feature based geometric matching. This VI needs to be setup for the parameters before running. There are various parameters which need setting for the proper functioning. The extraction mode has to be set to Uniform Regions so that the VI makes assumptions that the regions are uniformly pixelated and the result tends out to extract more uniform curves from the image. A Normal mode would not allow the VI to make any assumptions of the uniformity of the object and hence would result in less accurate results.

The edge filter size is set to the Contour Tracing option. This would enable the execution to extract the contour features of the image. Though it increases the processing time of the comparison, it yields much accurate results. For a faster comparison, the edge filter may be set to the Fine option. This would reduce the time taken, but would decrease the accuracy of the comparison. Contour tracing would provide the best results for the extraction of the features of a human face. The option of Min. Length can be set to define the minimum length of the curve that must be valid to be included in a comparison

Keeping the high values of this option would yield inaccurate results as many important features of the facial part would be ignored [9]. It has been therefore kept to a minimum value of 10. The Max Endpoint Gap option has been set to the value of 10 so that a gap between two curves greater than that length would indicate that it's an open curve. This defines two separate facial features of different values. The rest of the values have been kept unaltered from their default values.

In the IMAQ VI for the learning of Geometric pattern, the above stated setup is fed. The other parameters also need to be set accordingly. The origin offset needs to be set to the values of $(0,0)$ so that the centre of the image template is not shifted. This would treat the centre of the template as the basic point for the comparison with the images under testing. The Learn mask option has been set to 0 so that the curve parameters already defined in the previous VI are considered. It would maintain its default behavior and would correlate the image with the template accordingly. The template out from this VI can be taken so that it provides a frame of reference for the next VI. The template does not undergo any changes from this output, but a sequential representation helps in the smooth flow of the steps of the system. The rest of the values of the VI are kept to default.

\subsection{Matching Of Geometric Patterns}

The next step for the system is the matching of the geometric pattern of the template and the image. For this, the reference of the image as well as the template image is fed into the VI. This provides both the input image as well as the template for the comparison. If the ROI is drawn on the image inspection area by the user, only that part of the image would be inspected. The rest of it would be ignored. If no ROI is drawn, it would include the whole of the image into its inspection. In this system, the whole of the image needed to be inspected, so no ROI needed to be drawn.

\subsection{Match Score Optimization}

An important feature that needs to be set in this VI is the Match Score. This is considered as the minimum score that the two images under comparison must have in common so that the match is considered as valid. The value ranges from 0 to 1000 . A high value indicates that the images would be compared with more precision while the low score indicates that the lesser amount of precision would be employed, generally, a score between 800 to 950 gives out the best results. Facial features vary real-time, and hence keeping a high score would not give accurate results at all. It would be optional to set the 'minimum number of image matches requested' option because if the found number of matches are lesser than that value, it would give out no result. The output indicator of the found number of matches would indicate the number of exact matches of the template found in the database.

$$
\text { match score } \text { out }=\frac{\text { matched pixels }}{\text { total pixels in ROI }} X 1000
$$

Equation (2) represents the match score calculation criteria. A factor of 1000 has been taken to avoid decimal values and provide a higher precision to the checking of the image. The matching employed here is the pixel matching, hence the output is taken from the Matches (Pixel) terminal of the VI. The output from this terminal has been directly fed to the next VI. No indicators needed to be displayed as there has been no requirement of the data of the match since the ROI match worked properly.

\section{RESULTS AND DISCUSSION:}

The system has been tested using the various types of images, starting with the images of simple type 2 dimensional features, which had just a few or no contour features. These images were easy for the system to compare, hence the system showed no problems pertaining to these comparison. Then the image level is increased to the complex images, consisting of human faces or complex contoured features. Final matching has been done using the collection of images in the database with the predefined image template samples.

\subsection{Testing with simple Images:}

Testing with the simple images yielded the fully correct results. These images had no features related to contours but just to edges and spaces. These images were comparatively much easier for the system to recognize as there was no need of feature extraction.

Table 1 shows the use of the library images from the LabVIEW examples for the purpose.

The desired match score was tested to be set to very high levels and it gave positive results even at such high desired score. A zero output score indicates that there was no match corresponding to that image template at the particular desired score. Hence the score had to be decreased to some extent to relax the matching criteria. This gave the positive results once again.

The results were as follows: 
Table 1: Showing the image match results for simple images

\begin{tabular}{|l|l|l|}
\hline $\begin{array}{c}\text { Instances } \\
\text { of } \\
\text { template } \\
\text { in Image }\end{array}$ & $\begin{array}{c}\text { Desired Match Score } \\
\text { (out of 1000) }\end{array}$ & $\begin{array}{c}\text { Output Match } \\
\text { Score } \\
\text { (Out Of 1000) } \\
\text { (rounded off to } \\
\text { the nearest tens) }\end{array}$ \\
\hline 1 & 500 & 850 \\
\hline 2 & 600 & 920 \\
\hline 3 & 600 & 820 \\
\hline 4 & 600 & 840 \\
\hline 4 & 700 & 840 \\
\hline 4 & 850 & 0 (no match found) \\
\hline 4 & 835 & 840 \\
\hline
\end{tabular}

Fig. 2 shows the match results of the images taken from the sample gallery of the LabVIEW library. This process was carried out by using the rotation and scaling options while execution. Hence, the rotated and dissimilar proportion images have been included in ROI. All the instances of the images have been successfully recognized in this process.

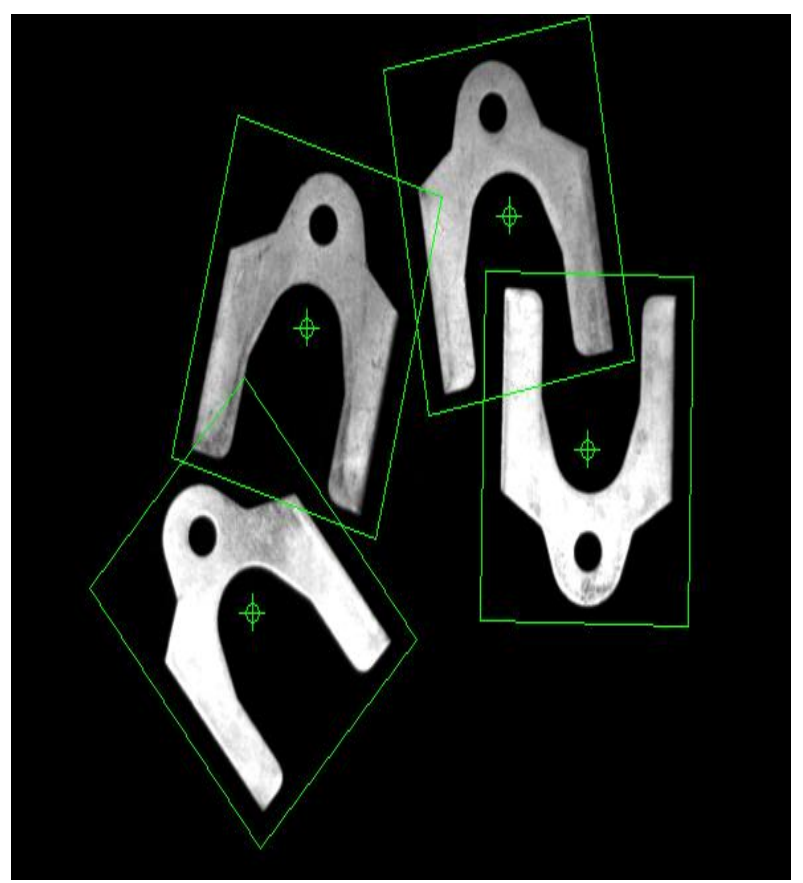

Fig 2: Simple images being compared and ROI been drawn around each instance of match.

\subsection{Testing with Facial Images:}

After the successful match of the simple images, the images with facial features have been taken for testing of the system. The facial image templates have been extracted using the gold template technique, which enabled to extract the images properly along with all their features. In addition to this, the feature based and the edge based template extraction was already enabled. The images taken were mostly the simple expression images with clear faces and neatly combed hair so that the image comparison could be facilitated. It was hence, easier to compare the images.

Fig. 3 represents a successful image comparison of the image been done, where the ROI has been drawn around the face of the person in the image whose template was compared from the database. This image taken is simple one which has just a single face. In other testing times, also more than one facial image has been taken into the execution of the system. The tabulated data is collected for the result analysis. It is noted that the performance of match declined steadily with increase in the desired score as well as the number of faces in the image database. It has to be done in such a way that the desired score could be balanced to keep the system accurate as well as free from ignorance of important features of the map.

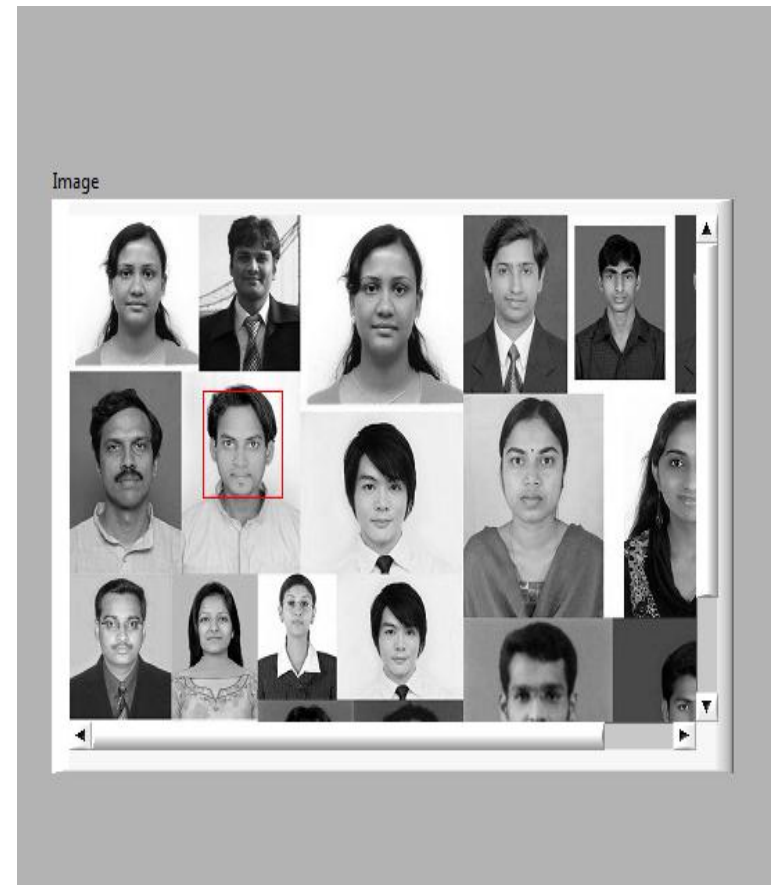

Fig 3: Showing the ROI been drawn around the face.

Table 2 represents the data from the image comparison when there are human faces present in the pictures. It represents the total number of faces in the image database $\mathrm{v} / \mathrm{s}$ the faces present in the template. On a higher desired match score, the probability of the image match is lesser as it demands the more complex comparison which would ignore many minor unmatched aspects and hence would not recognize either all or some of the faces. Hence the desired score has to be decreased to certain levels, step by step, to enable it to recognize the facial features.

Table 2: Data for facial recognition

\begin{tabular}{|l|l|l|l|}
\hline $\begin{array}{c}\text { Total Faces } \\
\text { In Image } \\
\text { Database }\end{array}$ & $\begin{array}{c}\text { Desired } \\
\text { Match } \\
\text { Score }\end{array}$ & $\begin{array}{c}\text { Whether } \\
\text { Recognised } \\
\text { All The } \\
\text { Faces From } \\
\text { Template }\end{array}$ & $\begin{array}{c}\text { Output } \\
\text { Number Of } \\
\text { Faces } \\
\text { Recognized }\end{array}$ \\
\hline 1 & 950 & NO & 0 \\
\hline 1 & 900 & YES & 1 \\
\hline 2 & 950 & NO & 0 \\
\hline 2 & 850 & NO & 1 \\
\hline 2 & 800 & YES & 2 \\
\hline 3 & 800 & YES & 3 \\
\hline 4 & 700 & NO & 0 \\
\hline 4 & 600 & YES & 4 \\
\hline 5 & 700 & NO & 2 \\
\hline 5 & 650 & NO & 3 \\
\hline 5 & 600 & YES & 5 \\
\hline
\end{tabular}


Hence Table 2 shows that for a higher number of faces present in the image database, a lower score of the desired match has to be kept. This would indeed make the match less secure, but would facilitate avoiding failures in match. For a higher number of faces in the image database, the matching would be slower and would gradually consume higher amount of memory.

\section{CONCLUSIONS}

Considering the results inferred from the testing and analysis of this system, the following has been observed:

- Grayscale images are best suited for comparison. They have the best distinct features for the feature extraction and hence give best results while comparison.

- Golden template extraction technique is best suited for Image Recognition systems which employ template matching. Golden templates can include a good variety of features for the purpose, imbibing the minute of the facial or image features.

- For facial recognition, the image pixel matching must be initiated from the origin of the axes. This helps in perfect matching as well as scans the whole image for the number of instances present in the input image.

- Simple images with no or minute contour features are easy to recognize. To save memory space for the system, only the edge detection technique may be chosen for the images which have no contours.

- The higher is the Desired Match Score, the higher is the matching precession of the image and the lower is the probability that it would match with the image database. A higher score increases the match difficulty and hence there may be a source of error.

- Match score has to be optimized in such a way that it makes a perfect trade-off between the security levels as well as with the comparison level.

\section{REFERENCES}

[1] J. M. Buhmann, J. Malik, and P. Perona, "Image recognition: Visual grouping, recognition, and learning," Proc. of the National Academy of Sciences, U.S.A, vol. 96, no. 25, pp. 14203 - 14204, Dec. 1999.

[2] Pedro F. Felzenszwalb and Daniel P. Huttenlocher, "Pictorial Structures for Object Recognition," International Journal of Computer Vision, vol. 61, no. 1, pp. 55-79, Jan. 2005

[3] David G. Lowe, "Distinctive Image Features from ScaleInvariant Keypoints, " International Journal of Computer Vision, vol. 60, no. 2, pp. 91-110, Jan. 2004.

[4] Gary W. Johnson and Richard Jennings, LabVIEW Graphical Programming, $4^{\text {th }}$ Ed, pp. 2-9, Jan. 2006.

[5] Thomas Klinger, Image Processing with LabVIEW and IMAQ Vision, Prentice Hall, 2003.

[6] Gyu-Hee Park, Hwa-Hyun Cho, and Myung-Ryul Choi, "A Contrast Enhancement Method using Dynamic Range Separate Histogram Equalization," IEEE Trans. on Consumer Electronics, vol. 54, no. 4, pp. 1981-1987, Nov. 2008.

[7] Shan Du, "Adaptive Region-Based Image Enhancement Method for Robust Face Recognition Under Variable Illumination Conditions," IEEE Trans. on Circuits And Systems For Video Technology, vol. 20, no. 9, pp. 11651175, Sept. 2010

[8] A. Samal and P. Iyengar, "Automatic recognition and analysis of human faces and facial expressions: A survey," Pattern Recognition, vol. 25, no. 1, pp. 65-77, Jan. 1992.

[9] R. Chellappa, C. L. Wilson, and S. Sirohey, "Human and Machine Recognition of faces: A survey," Proc. IEEE, vol. 83, no. 5, pp. 705-740, May 1995.

[10] M. S. Bartlett, J. R. Movellan, and T. J. Sejnowski, "Face recognition by independent component analysis," IEEE Trans. on Neural Networks, vol. 13, no. 6, pp. 1450-1464, Nov. 2002.

[11] H. Hajj, T. Nguyen, and R. Chin, "On multiscale feature detection using Filter banks," Proc. IEEE on Signals, Systems and Computers, vol. 1. pp. 70-74, Nov. 1995. 